\section{Violencia, espacio y vida cotidiana en la guerra mexicana contra las drogas: un análisis de Tijuana}

\section{Violence, urban space and everyday life in the Mexican War on Drugs: Tijuana as a case Study}

\author{
Héctor E. Bezares Buenrostro*
}

\section{Resumen}

La espacialidad de la Guerra contra las drogas en México ha sido escasamente estudiada. Bajo ese supuesto el autor de este artículo propone

Universidad Nacional Autónoma de México. Facultad de Ciencias Políticas y Sociales. Av. San Lorenzo 750 Mz.7 E 5 D 201 Col. San Nicolás Tolentino. Del. Iztapalapa, Ciudad de México, México, cp. 09850 hectorbezaresb@gmail.com una ruta analítica para examinar el modo en que el espacio urbano en la ciudad fronteriza de Tijuana ha sido re-significado y recompuesto bajo su representación como un sitio inseguro, violento y peligroso en el contexto de la estrategia antinarcóticos mexicana. Dicha re-presentación coincide con la expansión urbana y demográfica de la ciudad, así como con su inserción en los circuitos continentales de distribución de drogas. Tras inspeccionar esos procesos y sus efectos sobre la economía simbólica de la ciudad, el artículo indaga las consecuencias que esto ha tenido sobre la elaboración de las identidades políticas de sus residentes. Determinando formas de vulnerabilidad extrema de los habitantes de Tijuana, la Guerra contra las drogas ha organizado, sobre todo, un espacio en que el ejercicio de formas de violencia ilimitadas es constante y dentro del cual la vida política de la ciudad ha sido reducida al máximo, con graves consecuencias para el ejercicio de los derechos de sus habitantes.

Palabras clave: Tijuana, guerra contra las drogas, México, espacialidad, violencia

\footnotetext{
Abstract

The following paper explores the spatiality of Mexico's war on drugs by developing an analytical frame to examine urban space in Tijuana, a Mexican northern border city. Tijuana has been signified and construed as an unsafe and violent place within the context of the Mexican antinarcotics strategy. Its construction as a dangerous space coincides with its urban and demographic expansion, as well as its connection with the global circuits of drug distribution and the implementation of the war
} 
on drugs within the city. By representing it as an unsafe space, it has been authorized the exertion of unlimited violence upon its users and inhabitants by both state and private agents producing forms of extreme citizen vulnerability. As a consequence, both the political life of the city and its citizens' rights have been reduced to their bare minimum. Building on the analysis of Tijuana's symbolic, demographic and urban configuration, the consequences these processes have had upon the production of the city's residents' political identities are explored. By delving into the microphysics of the geographical imaginations on criminality and drugs in Tijuana, this paper contributes to the analysis on the ways in which the exertion of violence within the war on drugs is linked to the variegated forms urban space is interpreted by both citizens and authorities.

Key words: Tijuana, War on Drugs, Mexico, spatiality, violence.

\section{En Tijuana: una introducción}

En Julio del 2016 entrevisté a Fernando por segunda vez, para precisar algunos detalles de su vida en Tijuana y averiguar la medida de la destrucción operada en la ciudad, como consecuencia tanto del reciente incremento en los índices de criminalidad, como de los operativo policiaco-militares implementados en el contexto de la declaración de Guerra contra las drogas en México (Astorga 2005; Rochlin 1997). Declarada a comienzos del 2007 por el presidente Felipe Calderón Hinojosa, del conservador Partido Acción Nacional, a ella sucedió la duplicación del presupuesto militar, el inicio de los operativos policiaco-militares para pacificar determinados centros urbanos, sobre todo en la frontera norte, y el incremento de la letalidad homicida a lo largo del país, con particular intensidad ahí donde estas intervenciones tuvieron lugar (Felbab-Brown, 2011; Morales, 2012). Una de ellas en la ciudad de Tijuana.

En esta segunda entrevista, Fernando reitera el motivo de la desmemoria. Insiste en que no recuerda la fecha exacta, pero especula sobre ella al recordar la llamada y posterior visita de aquel hombre que vino hasta su negocio a ofrecerle servicios de protección. Pudo haber sido a la mitad del 2006 o en el 2005, como sugiere. Piensa y regresa en el tiempo mientras cierra los ojos y evoca el tiempo ausente, pero me dice que de cualquier forma es irrelevante saber el momento exacto. Sobre todo porque, musita, no alcanza a distinguir una diferencia mayor con los años que estaban por venir y ese momento específico. La masa de acontecimientos y eventos dibujan un continuo que ha debilitado la capacidad de discernir entre pasado y presente de la mayoría de los residentes locales y Fernando no es la excepción.

El motivo de la visita que convoca el recuerdo de Fernando es simple: un ofrecimiento para cuidarlo a él y a su familia, frente al avance de la inseguridad en la ciudad. El hombre que le visita es un viejo conocido de un centro de rehabilitación, en el que Fernando estuvo internado tras un problema de adicción al cristal, y al que no recuerda haberle dado su teléfono ni dirección. ${ }^{1}$ La visita siguió a una serie de llamadas a través de las que aquél hombre ofreció con insistencia sus servicios; 'no fuese a pasarle algo a él y su familia de no aceptarlos, pus ya ves cómo están las cosas de culeras

El cristal es una metanfetamina de gran popularidad en la frontera. 
por aquí', escuchó que le decía aquel individuo a través del auricular. ${ }^{2}$ Ambas, las llamadas aunque sobre todo la visita, confirmaron a Fernando que las cosas podían ir peor y que la ciudad, por caso, siempre podía descender un nivel más en su capacidad para violentar a sus habitantes.

Por aquellos años (entre 2005 y 2007), Tijuana entró en una espiral ascendente de violencia incontenible, que justificó y condujo a la entrada de la Policía Federal y el Ejército al final de aquél último año. Localización de una de las primeras operaciones de pacificación urbanas conducidas por Felipe Calderón, Tijuana estableció la ruta operativa sobre la que la supervisión de centros urbanos estratégicos, primordialmente localizados en la frontera norte mexicana, habría de sucederse en los años siguientes: intervenciones policiaco-militares espectaculares, supresión de las fuerzas de policía municipales, militarización de la seguridad pública e incontables retenes para regular la circulación de sus habitantes (Morales 2013).

En los años previos a dicha intervención amigos y conocidos de Fernando fueron objeto directa o indirectamente de alguna forma de agresión: secuestros, robos y asesinatos. Inscritas estas agresiones en un círculo más amplio de balaceras constantes, ejecuciones a la luz del día, cuerpos mutilados, secuestros y enfrentamientos entre grupos de narcotraficantes, nadie en su círculo de conocidos pudo evitar los estragos

El término culero refiere en este caso a la descomposición de la ciudad y puede asumirse que significa literalmente que las cosas están mal. La conservación de los términos y modos usados originalmente por mis informantes, tiene como finalidad realizar una transcripción precisa de su posición en el contexto más amplia del funcionamiento de la ciudad. De acuerdo a su aparición en el texto, y a través de notas al pie de página, su significado será explicado según sea el caso. provocados por la violencia, de los cuales los mayores eran la incertidumbre y el miedo. En ese sentido, la forma anónima de esa violencia y la incapacidad, incluso policiaca de identificar sus fuentes concretas, multiplicaron el miedo. Todos, sin embargo, acabaron, autoridades incluidas, refiriéndola como una prolongación de la disputa entre bandas de narcotraficantes por el territorio de la ciudad; o lo que en la jerga periodística mexicana ha sido llamada la lucha por la plaza. (Shirk \& Wallman 2015)

La visita de aquel hombre para ofrecerle seguridad, constituyó para Fernando la metáfora perfecta de la incertidumbre que aquel estado de cosas encarnaba:

\begin{abstract}
Trabajé toda la vida para levantar mi negocio. Un día, casi de la nada, esos güeyes (los narcos) pasaron de matarse entre ellos, a secuestrar y matar a gente que no estaba en pedos de drogas. Ya sabes, el pedo de la plaza, que los del Chapo contra el cartel de Tijuana, ve tú a saber qué estaba pasando. El día que ese cabrón se apareció por la agencia ofreciéndome protección supe que tan mal podían estar las cosas. Si no podías confiar en la policía y si los malandros estaban por todas partes, si los policías mismos eran malandros, pus eso, no estabas a salvo. Si no le pagaba por la protección el pedo iba a ser que se las iba a cobrar conmigo y ponerme con algún malandro o con la policía para sacar unos pesos. Al final no le di ni madres, pero no dormí bien como seis meses nomás de estar pensando en cuando me iban a chingar a mí también. Mis hijos, que ya vivían en Phoenix estudiando la prepa, no vinieron todo ese año a Tijuana, yo cambié cerraduras, puse rejas en las ventanas y dejé de salir lo más que pude. ${ }^{3}$
\end{abstract}

\footnotetext{
El término güey es propio del habla cotidiana en México y es utilizado de manera ofensiva para referirse a cualquier persona, sin distinción de género. Alude inicialmente a alguien tonto, pero que por su uso y familiaridad, ha devenido en una forma coloquial de designar a extraños o conocidos. El término equivalente en inglés que puede facilitar su comprensión es el de dude. Pero en este caso, refiere a la existencia de un problema; malandro es un término hoy de uso generalizado para designar a alguien asociado a la vida criminal; cabrón en México es un término derogatorio que se utiliza para designar indistintamente alguien cuya fuerza se estima sobrepasa la propia.
} 
Convertida en la trama dominante de las explicaciones sobre la organización de la violencia y la inseguridad en la ciudad, así como de sus efectos correlativos sobre la vida cotidiana de los habitantes de Tijuana, la lucha armada entre narcotraficantes terminó por explicar todo: el silencio de las autoridades, la sospecha justificada respecto a los vecinos recién llegados al barrio o frente a los extraños en la calle, el auto-encierro y el consecuente abandono de los espacios públicos. En ese sentido, la significación de la ciudad como un espacio peligroso y violento asociado al tráfico de drogas, resultó también en la clasificación del espacio urbano en zonas seguras en contraposición a zonas violentas e inseguras, así como de sus habitantes de acuerdo con la posición que guardan con relación a dicha distribución espacial de la inseguridad. Este proceso de largas y duraderas consecuencias habilitó la infraestructura imaginaria y afectiva, sobre la que eventualmente se hizo posible la ocupación de la ciudad en la administración de Felipe Calderón. Con graves consecuencias para la vida de la ciudad, su organización, uso y disfrute, así como para el modo en que formas de ciudadanía diferenciadas espacialmente han sido construidas, es el análisis de esa trama y de la reconfiguración de los espacios habitados por los tijuanenses, el objeto central de este artículo.

Principalmente a través de información obtenida de una investigación de carácter etnográfico (entrevistas y observación participante), llevada a cabo en Tijuana en 2016 durante 4 meses, este artículo analiza la codificación del espacio de la ciudad como un sitio inseguro. La investigación se concentra en los años previos a la ocupación y pacificación militar de la ciudad. Específicamente, el artículo analiza las consecuencias que esa codificación de la ciudad tuvo sobre la organización y diferenciación del espacio público, así como para la clasificación de sus habitantes de acuerdo a criterios de inseguridad, riesgo e incertidumbre.

Como fue mencionado arriba, el análisis se enfoca sobre el periodo anterior a la pacificación emprendida por el ejército y policía federal mexicana en 2007. En dicho periodo, de inicio incierto, pero que los entrevistados sitúan entre 2003 y 2006, rupturas importantes ocurrieron en el orden simbólico y material de la ciudad. Es ese periodo de incertidumbre, el que autoriza la intervención policiaco-militar de la federación en el orden local, al convertirla en una necesidad ante lo que se vive en el terreno como una crisis profunda de inseguridad. Su análisis permitirá comprender la emergencia de formas novedosas de representación y uso de la ciudad, a propósito de la expansión de la inseguridad y la violencia asociada al narcotráfico. Más importante aún, habilitará nuestra compresión de algunas de las condiciones que hicieron posible el ejercicio de la violencia estatal, en la forma de la posterior ocupación policíaco-militar de la ciudad.

Primero, será analizado el modo en que históricamente el espacio urbano en Tijuana fue construido como inseguro, en el contexto más amplio de su histórica vocación turística y su relación con Estados Unidos. Luego entonces, serán trazados los puntos de inflexión y fractura, de los que emerge tanto la reconfiguración de Tijuana como un espacio de violencia y alta peligrosidad, como la modificación de las percepciones de sus residentes, con efectos importantes para la organización de su identidad política y ejercicio de su ciudadanía. De ese modo, atendiendo a las representaciones emergentes sobre la ciudad de Tijuana y a la 
clasificación de sus espacios como inseguros, daremos cuenta de cómo la geografía de la ciudad ha sido producida en la intersección entre la forma urbana, su imaginación y la inseguridad rampante.

Tomar como objeto de análisis este proceso de configuración y organización del espacio urbano, es fundamental para el entendimiento de algunas de las condiciones que hicieron posible el despliegue policiaco y posterior militarización de la ciudad. Dicha militarización como es analizado en otra parte (Bezares 2018), sigue las líneas de fractura abiertas por la inseguridad, pero sobre todo las distinciones geográficas del trazo urbano, organizadas alrededor de lo que Pilar Caldeira (2000) llama la conversación del crimen. Es decir, los modos discursivamente organizados y autorizados, a través de los que las diferencias en el espacio urbano son organizadas y a través de las que se discrimina entre lo que es seguro e inseguro. En este sentido, a la violencia producida por el aumento de la inseguridad en Tijuana le siguieron formas de auto-segregación, confinamiento, así como discursos ad hoc, que hicieron posibles formas de obediencia y anuencia colectiva, que en última instancia autorizaron la posibilidad de la eventual pacificación de la ciudad.

El artículo se encuentra primordialmente en diálogo, con una discusión académica que ha puesto su atención sobre la manera en que los espacios de la vida cotidiana, se entrelazan a la reproducción de circuitos de violencia e inseguridad, asociados al gobierno de los individuos (Glück \& Low 2017; Gregory, 1994 2011; Knowles 2000; Legg 2007; Rose, 2004). Específicamente, el análisis construye sobre una literatura que ha tomado como objeto de estudio la transformación del espacio urbano en Latinoamérica (Davis 2017; Glebbeek \& Koonings 2015; Koonings \& Kruijt 2007; 2009), la proliferación de violencias asociadas a dicho crecimiento, así como la emergencia de formas de segregación socio-espaciales constitutivas de formas de distinción y conflicto. Adicionalmente, problematiza los ejercicios teórico-conceptuales que han dominado el análisis reciente de la guerra contra las drogas, así como la escalada de violencia que le ha acompañado. De ese modo, el artículo proporciona un contrapunto conceptual e interpretativo a las visiones dominantes sobre la guerra. Primero, proponiendo un análisis de las micro-espacialidades que orientan la percepción y organización de la violencia en Tijuana. Segundo, ampliando la evidencia disponible, a través de la recuperación de los relatos de los habitantes de Tijuana sobre la inseguridad y el miedo emergentes en el contexto de la intensificación de la criminalidad en dicha ciudad. Así, es abierta 'desde abajo' una puerta de acceso a las condiciones socio-espaciales, en que los tijuanenses se encuentran inmersos y que ha sido escasamente explorada en la literatura sobre el tema. Dichas condiciones, articulan los flujos discursivos dentro de los que los habitantes de la ciudad se posicionan, en las constelaciones de poder que organizan la vida de esta ciudad (Knowles 2000). De acuerdo con lo anterior, el artículo contribuye inicialmente a la literatura sobre la guerra contra las drogas, al ampliar la evidencia disponible sobre la articulación espacial de la violencia asociada a dicha estrategia. En seguida, contribuye también al ampliar nuestro entendimiento sobre la operación del miedo y la distinción, como organizadores de los espacios públicos y privados, generando condiciones adversas para el ejercicio de la ciudadanía y la democracia (Caldeira 1996). 
Este trabajo procede de acuerdo al siguiente orden. En la sección subsiguiente, son discutidas las principales líneas interpretativas en torno la guerra contra las drogas en México, considerando porprincipiolaabundanciadeenfoquescentrados en los riesgos para el estado. El apartado tiene como propósito, a través de una revisión de la literatura relacionada, resaltar las ventajas de un enfoque que analice el espacio urbano y la vida cotidiana, así como situar este trabajo en el contexto de la discusión sobre la guerra contra las drogas en México. A continuación, el análisis problematiza esta visión, refiriendo los debates sobre la construcción del espacio, la organización de la ciudad y la proliferación de la violencia, como punto de partida para enlazar la investigación sobre la guerra contra las drogas con la producción del espacio. Ello permitirá el examen de la intersección entre la organización material e imaginaria de la ciudad, así como el desplazamiento de los referentes que estructuran la economía política de su funcionamiento, en el contexto de la proliferación de la violencia y la guerra contra las drogas. Especial énfasis es hecho en la relación entre la ordenación del espacio, la conformación de identidades y los usos posibles y autorizados de la ciudad. En la siguiente sección de este texto es discutida la configuración histórica de la ciudad de Tijuana, su ordenación como un espacio orientado para la provisión de satisfactores ilegales para Estados Unidos, y la resultante identificación de su geografía como un espacio de riesgo. Esto ha producido y especificado formas concretas, de lo que Glebbeek y Koonings (2015) Ilaman ciudadanía subjetiva. Dichas formas de ciudadanía subjetiva, refieren posibilidades y usos concretos del espacio urbano, así como el acceso y disfrute de derechos políticos (sección 4). Finalmente, en las secciones subsiguientes es analizada una de estas formas de ciudadanía subjetiva, en su relación con la configuración de los espacios de la ciudad como altamente inseguros: el ciudadano vulnerable.

\section{Explicaciones sobre la guerra contra las drogas en México}

En esta sección son abordadas algunas de las vertientes de las discusiones académicas en torno a la guerra contra las drogas en México. Sobre todo aquellas establecidas en torno a la protección y defensa de la seguridad del estado mexicano. Sus principales argumentos son señalados, así como la ausencia relativa de investigaciones con respecto a los aspectos micro-políticos y espaciales de dicha guerra. Aspectos que conciernen tanto a la reconfiguración de las ciudades bajo la lógica de dichas intervenciones policiaco-militares, como a sus efectos sobre los espacios de la vida cotidiana, y el modo en que sus residentes han sido afectados.

Los enfoques recientes sobre la guerra contra las drogas en México se aproximan predominantemente a ésta, desde la presunción de la división fundamental entre crimen organizado y estado. Desde un nivel macropolítico, que concierne al análisis a una escala nacional yestatal, estos enfoques intentan explicar los patrones y dinámicas de violencia (Molzahn et. al. 2012; Morales 2013; Shirk 2014), señalando el incremento de la violencia con relación a la intensificación de los homicidios, su localización en el espacio y la relación entre este incremento y la más amplia transformación de los patrones transnacionales de producción e intercambio de drogas (Paley 2014). Así mismo, se ha abordado la organización del crimen organizado, enfocándose primordialmente en 
las características y perfil de las organizaciones criminales a que se enfrenta el estado (Bunker \& Sullivan 2010; Campbell 2010; Womer \& Bunker 2010). También han sido examinadas las estrategias estatales de contención, intervención y combate emprendidas en la etapa más reciente de la guerra contra las drogas en México (Astorga \& Shirk 2010; Kenny \& Serrano, 2012; González 2009; Morales 2013; Serrano 2012). Desde una perspectiva más crítica, ha sido evaluada la relación entre el reciente índice de letalidad asociado a la expansión del crimen organizado, y la intensificación de las operaciones punitivas emprendidas por el Estado (Astorga, 2015; Escalante, 2011).

En estos estudios sobre la guerra contra las drogas en México, tanto las organizaciones criminales como el Estado son conceptualizados en una lucha perpetua. Dicha confrontación, explican, es agudizada por la combinación exacerbada de violencia y corrupción, lo que ha derivado en la desestabilización del estado, la fragilidad de la seguridad nacional y la desestabilización de la vida democrática del país. Estos estudios aportan importantes evidencias y reflexiones, sobre las dimensiones nacionales de la violencia emergente en la guerra del estado contra las organizaciones criminales. Sin embargo, poco explican sobre los contextos socio-espaciales en que la guerra contra las drogas ha sido implementada, sus efectos en el modo en que los individuos son gobernandos en dichos contextos, así como las amplias transformaciones que las ciudades en que ha sido instrumentada han experimentado. Inversamente, estas investigaciones dicen poco sobre la manera en que los espacios intervenidos por la guerra contra las drogas han afectado su implementación, curso, diseño, o suposiciones fundamentales.
En este sentido, estos enfoques macropolíticos poseen una limitada tracción analítica en lo que respecta a los aspectos cotidianos de la guerra contra las drogas, así como en la manera en que cuerpos y espacios se entrelazan, en la articulación de los efectos concretos de la violencia de la guerra sobre los ciudadanos mexicanos. En este caso, es omitido el examen del modo en que la guerra contra las drogas, se materializa en la vida cotidiana de los usuarios de los espacios en que ésta tiene lugar. Estas limitaciones tienen como consecuencia un estrechamiento teórico que dificulta el análisis de los vínculos entre lo local y lo transnacional; de las maneras en que lógicas extra-nacionales son confrontadas y asimiladas a través de configuraciones socio-políticas alternativas al Estado; y de cómo la guerra ha modificado el modo en que la ciudadanía es configurada espacialmente y finalmente hecha posible. Ello disminuye nuestro entendimiento sobre los modos concretos de realización de la guerra, así como sus efectos sobre la conformación de las relaciones socio-espaciales que hacen posible interacciones políticas específicas entre los individuos.

En contraste con ello, una literatura también reciente ha abordado de forma indirecta la constitución espacial de la guerra contra las drogas. Boyce et al. (2015), proponen el entendimiento del uso de metaforas espaciales de las autoridades mexicanas, instrumentos ideológicos que propician la demarcación del territorio para hacer posible la visualización del mismo como un espacio en guerra y posibilitar así su ejecución. Campbell (2009) por otra parte examina la constitución de un espacio, "the drug war zone", en que los discursos y prácticas de las fuerzas del orden estatal y los criminales confluyen. En ese espacio, las 
características materiales de las geografías realmente existentes, se entremezclan con las cualidades ficticias atribuidas, en este caso, a las zonas fronterizas de México, creando lógicas simbólicas y rituales distintivas, orientando la acción de aquellos individuos que participan de su existencia. Melissa Wright $(2011 ; 2013)$ examina, por otra parte, el efecto que la guerra contra las drogas ha tenido sobre la constitución del espacio urbano, las estrategias de gentrificación y la supervisión policiaca de Ciudad Juárez, en los años que siguieron a las intervenciones militares sobre dicha ciudad. Así mismo, Mendoza (2008) en un trabajo de naturaleza etnográfica sobre el tráfico de drogas en una comunidad semi-rural en el norte de México, analiza, la organización del narcotráfico y la vida cotidiana de sus habitantes.

No obstante, en términos de la exploración de esta guerra, poco se ha dicho sobre los efectos cotidianos de las estrategias políciaco-militares de supervisión del espacio urbano, así como sobre las conexiones entre dichas estrategias, y las dinámicas anti-narcóticos continentales. De este modo, como consecuencia, se pasa por alto la contribución que un análisis socioespacial puede hacer, para comprender la manera en que la violencia de la guerra contra las drogas es prolongada geográficamente. Primero, explorando los modos prácticos en que los ciudadanos mexicanos hacen uso efectivo de sus derechos políticos en contextos urbanos, bajo las condiciones de violencia producidas por la estrategia antinarcóticos del gobierno mexicano. Y por último, analizando al nivel de la vida cotidiana, la expansión de las esferas de dominación estatales, así como la configuración de límites espaciales, actuales e imaginarios, dentro de los que los individuos organizan sus existencias e interactúan entre sí.
En ese sentido, este texto dialoga con una literatura que ha explorado la relación entre geografía y la formación de subjetividades políticas, para entender el ejercicio del poder, así como la manera en que el espacio geográfico es imaginado y representado, haciendo posible formas de intervención, violencia e identificación productivas de estructuras de exclusión y separación (Caldeira 1996, 2000; Chatterjee 2009; Gregory 1995; Rodgers 2006; Weizman 2007). A través de estos análisis se ha notado como en contextos urbanos contemporáneos, por acción de la violencia, han surgido formas novedosas de identificación y participación política, incentivado el conflicto, e incrementado la desigualdad en el acceso a bienes y servicios en la ciudad, con consecuencias desastrosas para la existencia colectiva e individual.

De acuerdo con lo anterior, este artículo propone una ruta analítica en dicha dirección. Considera para tal propósito, la exploración de las modalidades bajo las que, en la guerra contra las drogas en México, la agencia de los residentes de las ciudades intervenidas es formada a través de su posicionamieno y distribución en el espacio conformado por los discursos y prácticas antinarcóticos. Dicha orientación teóretica, permitirá el reconocimiento de los arreglos espaciales, a través de los que el poder se manifiesta. Es decir, cómo relaciones específicas de poder penetran la vida cotidiana, proyectando formas de ocupación, visualización y movimiento que posibilitan transformaciones en la forma en que individuos y comunidades se organizan, mueven y usan el espacio público.

De esa manera, señalando la importancia de lo cotidiano, lo local y lo corporal, en su relación con estrategias más amplias (transnacionales o nacionales) de control, la atención en la ciudad 
de Tijuana se concentra sobre dos procesos relacionados con la producción y ordenación de los espacios y los individuos en el marco de la guerra contra las drogas. El primero es la representación de dicha ciudad como un espacio criminógenico; y el segundo concierne al desarrollo por parte de sus habitantes, de un entendimiento y uso ad hoc del espacio urbano, con consecuencias profundas para la vida política de Tijuana. La observación de ambas dinámicas, permitirán al lector entender cómo la guerrra contra las drogas ha modificado el entendimiento de los residentes de esta ciudad fronteriza. Primero con respecto a sus límites, funciones y propósitos, afectando su constitución como sujetos políticos; al tiempo en que se ha hecho posible la ampliación de la actividad punitiva y disciplinaria del estado.

\section{Tijuana: orden urbano y perfil criminogénico}

Welcome to Tijuana

Tequila, sexo, marihuana Manu Chao

Esta sección argumenta que la historia y diseño urbano de Tijuana son claves para entender su inserción imaginaria en el entendimiento del público como un espacio peligroso y transgresivo. De su representación como un lugar de riesgos y liminar, se desprende a su vez la identificación de sus habitantes como potenciales transgresores. Posición reforzada desde el exterior de sus límites físicos, por estereotipos centrados principalmente en la historia de la ciudad como lugar de suministro de placeres fuera de la ley para los ciudadanos estadounidenses. Ello simplifica el gobierno de la ciudad, toda vez que al situar a sus habitantes en esa posición de transgresión potencial, pocas explicaciones tienen que darse por la proliferación de abusos policiacos, o el establecimiento de muros para la vigilancia de la frontera (Yeh 2017). De ese modo, la intervención policiaco-militar en la ciudad en 2007 con el propósito de pacificarla, se encontró con un terreno ad hoc a la lógica predicada por la guerra contra las drogas, y su idea de un territorio configurado como campo de batalla.

Tijuana se encuentra situada en los límites entre México y Estados Unidos, en el extremo noroeste de la frontera mexicana. La ciudad nace organizada económicamente en torno del suministro de bienes y servicios de bajo costo, para los pobladores estadounidenses, principalmente aquellos asentados en el puerto de San Diego. En el contexto de la prohibición del alcohol y la prostitución en Estados Unidos en las primeras décadas del siglo XX, Tijuana ofrece a los estadounidenses acceso ilimitado a estos bienes y servicios, engarzando su crecimiento a la provisión de productos, de otra forma ilegales en aquel país (Ruiz 1998).

Esta orientación económica primigenia, determinó también la composición de la forma urbana de la ciudad. ${ }^{4}$ De esa manera, y a diferencia del común de las ciudades latinoamericanas, organizadas alrededor de una plaza central, rodeada por los símbolos del poder religioso, económico y político, el trazo urbano de la ciudad de Tijuana se organiza alrededor de una franja horizontal que corre paralela a la línea fronteriza. Corredor comercial y de entretenimiento, alrededor de esa franja se irán progresivamente localizando los espacios industriales y residenciales (Enriquez 2007).

Replicada a lo largo de la frontera en otros poblados mexicanos, incorporados también informalmente a la órbita de consumo estadounidense (Enriquez 2007) 
Dispuestos de manera adyacente a la frontera y a lo largo de esa franja, la disposición de bares, farmacias, prostíbulos, cabarets y hoteles, permite el flujo constante de parroquianos y la circulación aparentemente ilimitada de dinero (Fig 1). También, por caso, la composición turístico-transgresora de la ciudad, definirá la reputación eidentidad de Tijuana comoun espacio sin ley en el que todo se encuentra permitido, tal y como sugiere una declaración de inicios del siglo XX de la Iglesia Metodista estadounidense: "Todo vale en Tijuana (...) El pueblo es la meca de prostitutas, vendedores de alcohol, apostadores y toda clase de fauna nociva americana (Citado en Berumen 2003: 178)".

Figura 1. Vista área de Tijuana

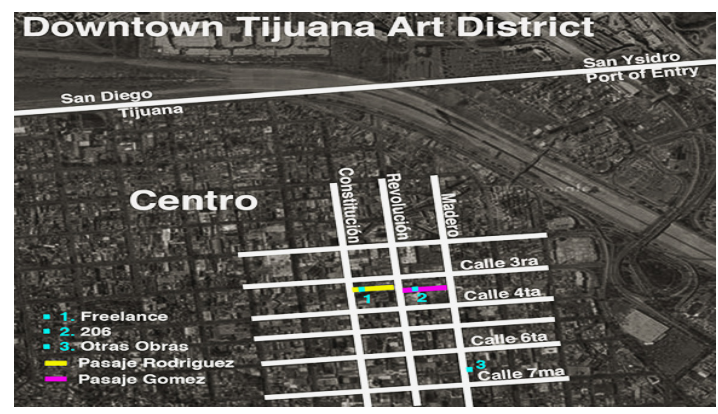

Fuente: Downtown Tijuana Art District, https://www.kcet.org/ shows/artbound/from-the-center-of-the-margin-contemporaryart-galleries-in-downtown-tijuana

Con profundas consecuencias políticas y económicas para la vida de la ciudad, sus habitantes explicarán también el valor simbólico de Tijuana, a propósito de esa posición liminal en la imaginación de sus usuarios:

M. Qué es Tijuana? Pus una ciudad de prostitutas, drogas y narcos. Pero también de gente trabajadora. Siempre ha sido así. Yo soy de Hermosillo y antes de casarme y venir aquí todos sabíamos eso. Nada ha cambiado desde entonces, y te estoy hablando de 1979. Mi mamá me dijo que estaba loca si quería venirme aquí cuando le dije que Raúl (su esposo) y yo nos vendríamos para acá. No es tan malo en realidad, porque sin los turistas hace mucho que la ciudad se habría acabado.

La observación que hace Marina de la naturaleza de la ciudad, replica los estereotipos sobre la misma, y explicaquees su no conformidad conlos estandares morales y legales estadounidenses, lo que le proporciona viabilidad y la hace atractiva contemporáneamente. Juan, otro habitante de Tijuana expande sobre esa observación y añade algunos otros elementos sobre su exuberancia:

Tijuana fue en su momento el centro de todo: la fiesta,
drogas, mujeres. Todo estaba aquí. Un día desperté después
de una semana de fiesta con tres pinches viejas a lado. Me
había gastado 200,000 varos. Fue quizá en el 2001 . Ya ni
sé. En esa época me metía un chingo de cristal y las cosas
se salieron de control. Pero así era en todos lados. Los
Arellano estaban arriba y Tijuana era el centro del universo. ${ }^{5}$

Las referencias que hace Juan, quien vende autos usados, ilustran sucintamente la actualización del status de Tijuana, que pasó a partir de los años 80 de ser exclusivamente un centro de atracción turístico a zona de tránsito de migrantes, drogas y proveedor de mano de obra barata para las maquiladoras estadounidenses y asiáticas ahí instaladas (Katsulis 2008). La época que él describe, sigue dos procesos que corren históricamente de forma paralela, y que afectaron la composición imaginaria y material de esta ciudad. Primero, la interrupción de la ruta del Caribe, tras la estrategia de interdicción emprendida por Estados Unidos en Sudamérica, entre finales de los ochenta y mediados de los noventa, para reducir el suministro de cocaína

Juan se refiere a 200,000 pesos que al tipo de cambio estimado de aquella época de más o menos 10 pesos por dólar debieron equivaler a 20, 000 dólares. 'Un chingo' se refiere a una gran cantidad, inestimable bajo una lógica estrictamente aritmética. Varos es un modo coloquial de llamarle al dinero en México. 
procedente de aquella región (Boyer 2001; Vulliamy 2011). Luego, en segundo término, a la liberalización comercial y financiera emprendida en la década de 1980 por el gobierno mexicano, tras su default crediticio de 1982 (Rochlin 1997). El primer proceso obligó a los intermediarios colombianos de los productores de cocaína, a utilizar el territorio de México como paso obligado a los Estados Unidos, empoderando a los intermediarios mexicanos. El segundo proceso generó las condiciones económicas indispensables, para la creación de un mercado favorable al narcotráfico, ante el agotamiento de fuentes de financiamiento externos para México en el contexto de la crisis económica de los años 80 (Rochlin 1997).

Ese tránsito histórico se introduce en el relato de Juan, a través de la abundancia de drogas y dinero, así como en la alusión que hace a los hermanos Arellano Félix, líderes de lo que en su momento fue conocido como el cártel de Tijuana. Ambas referencias hacen por lo tanto explícito el desplazamiento simbólico de la ciudad de un proveedor de prostitutas y alcohol, a puerto de salida de las drogas procedentes del sur, y lugar de excesos para sus propios habitantes.

La sobreposición, al estatus original de Tijuana como zona moralmente liminar, de su recientemente adquirida ubicación como una parte fundamental de la cadena de suministro de drogas a Estados Unidos, introdujo una tensión fundamental en la configuración imaginaria de la ciudad. A la ya dominante representación de la frontera México-Estados Unidos como un espacio anárquico "lleno de homosexuales, heroina, traficantes y padrotes" (Astorga 2003), se sumó su construcción como una parte central de las amenazas a la seguridad nacional de Estados Unidos.
Es en esa combinación entre la composición simbólica y material de Tijuana como un lugar sin ley, así como en el desplazamiento que se opera en términos de la geopolítica de las drogas estadounidense, que encontramos una emergente interpretación de la ciudad como un espacio de peligro a la que se necesita intervenir y purificar:

\begin{abstract}
J. Tijuana no es diferente a otras ciudades de la frontera. Peligrosa, sucia y llena de criminales. Pero si queremos empezar por algún lado para acabar con el problema del narcotráfico, es ahí donde teníamos que ir. Ahí se junta lo peor de todo: migrantes, drogas, prostitutas y narcotráficantes. Y eso contamina al resto. La idea es que llevaramos a la policía federal y que limpiaramos todo, desde el policía de a pie hasta la ciudad. No lo logramos pero por lo menos se intentó.
\end{abstract}

H. Y cómo identificaron ustedes quienes eran parte del problema, y quienes inocentes?

J. No hay forma de hacerlo, ni modo que le preguntes a cada uno. Así que agarramos parejo. (Juan, miembro de la policía federal)

Re-situada como una parte central del problema del país con el narcotráfico, y como extensión de la reconsideración del status de Tijuana, sus habitantes devinieron consecuentemente potenciales criminales, como sugiere este policía federal en el párrafo precedente. ${ }^{6}$ Alrededor de este desplazamiento de la identidad de la ciudad como un espacio peligroso asociado al narcotráfico, se organiza consecuentemente una clasificación emergente de sus residentes, y se establecen condiciones para su movimiento y uso de la ciudad. Dicho desplazamiento es, sin embargo, lento, y su consistencia material no del todo clara para sus propios habitantes.

\footnotetext{
La Policía Federal es un cuerpo policiaco semi-militarizado bajo el mando directo del presidente que nació en 1997, como parte de un decreto presidencial y con la intención de circunvenir las restricciones constitucionales que el ejército tiene para actuar en el restablecimiento del orden interno.
} 
En algún momento a la mitad de la primera década del siglo XXI, la ciudad adquiere no obstante un rumbo que permite constatar de forma factual la alineación entre el ritmo que toman los movimientos de individuos y cosas dentro de Tijuana, y su caracterización como un espacio inseguro propiciado por el narcotráfico.

La siguiente sección se ocupará, en consecuencia con lo anterior, de explicar cómo los residentes de Tijuana comienzan a situar esa alineación entre el desplazamiento de la identidad de la ciudad, la propia identificación de que son objeto como potenciales criminales, y la intensificación de la inseguridad en el espacio urbano.

\section{Espacio e identidad: Una ciudad de potenciales criminales}

De acuerdo consus habitantes, el funcionamiento de Tijuana antes de su inserción en una parte central de la cadena de suministro de narcóticos hacia Estados Unidos, y posterior envilecimiento dentro de los imaginarios geopolíticos de las drogas, tuvo siempre un elemento de inestabilidad, ambigüedad y tolerada criminalidad, en términos de los modos en que la ciudad era vivida e imaginada. En ese sentido, el tráfico de drogas ha sido permanentemente, una parte del paisaje criminal de la ciudad (Astorga 2005), normalizado entre la serie de actividades ilicitas constitutivas de la razón de ser, esencialmente turística, de la ciudad:

A. Yo llegué en 1982. Antes de venir acá lo que todo el mundo sabía de Tijuana es que era una ciudad de putas y drogas y nadie se asustaba. La diferencia con lo que sucede ahora es que es díficil saber ahora quién es quién. Sobre todo porque todo el tema de las drogas y las actividades ilicitas se concentraban en el centro, pero hoy que la ciudad ha crecido tanto y con todo el montón de gente que entra y sale de aquí, es díficil diferenciar. Con el agravante de que un día esta gente (los narcos) comenzó ya no sólo a matarse entre sí, sino a barrer con todos.

H. Cuándo comenzó a suceder eso?

A. No puedo decirte de un inicio claro, pero por ahí del 2006 las cosas se pusieron mal de verdad. Secuestros por todas partes, ejecuciones, desaparecidos. Mucha gente terminó yendose de Tijuana por eso. (Arturo, hombre de negocios)

Hay, sin embargo, un cambio cualitativo en los índices que miden el aumento de los crímenes y la inseguridad, que obliga a considerar su influencia en la reconfiguración del status de la ciudad, y su representación imaginaria hacia finales de los años 90 del siglo XX. La información estadística disponible señala con claridad que hacia finales de los 90 , la frontera norte mexicana se convirtió en un territorio particularmente violento. $40 \%$ de los crimenes que ocurrieron en el país, tuvieron lugar sólo en esta región (Escalante 2009; Romero 2012). En Tijuana, específicamente, después de 1994 el índice de homicidios pasó de 5 homicidios por cada 100, 000 habitantes, a 35.87 para el final de esa década. ${ }^{7}$ Además entre 1993 y 2012, de acuerdo con la Asociación Esperanza contra la Desaparición Forzada y la Impunidad -AEDFI- 1, 200 individuos fueron levantados por el crimen organizado (Almazán 2013). ${ }^{8}$

Aunque las explicaciones sobre las condiciones que hicieron posible esta intensificación de la violencia homicida, apuntan a la combinación entre el crecimiento demográfico, la expansión territorial y la falta de planeación urbana, éstas son insuficientes y más elementos son necesarios para determinar sus causas (Escalante 2009; 2011).

8 En la jerga de la guerra mexicana contra las drogas el levantón es una forma del secuestro, que sin embargo no busca cobrar ninguna suma de dinero para eventualmente regresar al individuo secuestrado con sus seres queridos. Es esencialmente una abducción cuya finalidad es desaparecer a la persona que ha sido secuestrada. En ese sentido guarda alguna similitud con la arbitrariedad de las dictaduras, aunque sin motivación ideológica alguna. En este caso la inducción del terror o la venganza, parecen fungir como motivaciones subyacentes. 
Este incremento de los homicidios y desapariciones, se reflejó en la vida cotidiana de Tijuana, a través de la sucesión cada vez más reiterada de crimenes espectaculares, la expansión de la incertidumbre ante la imposibilidad de determinar las razones $u$ orígen de dicho incremento, así como en la potencialización de la posibilidad de que cualquiera pudiese convertirse en víctima. La historia que Fernado refiere al inicio de este trabajo, coincide en el tiempo tanto con lo que Arturo manifiesta, así como con una serie más amplia de referencias recolectadas en el curso de mi trabajo de campo en esta ciudad. Todas apuntan en la misma dirección en dos sentidos convergentes: la observación sobre un desplazamiento radical en el funcionamiento de Tijuana, y la vulnerabilidad propia de las posiciones sus habitantes en un contexto en el que el discernimiento de las causas de la inseguridad, así como sus portadores, se torna impreciso y ambivalente. Por ello, es importante especificar el sentido del desplazamiento en la economía simbólica de la ciudad, operado tanto por su re-conceptualización como un centro de operación del narcotráfico, como por la proliferación de los homicidios. Al mismo tiempo en que ubicamos sus efectos sobre la identificación y clasificación de sus pobladores, colocando como eje de la reflexión la modificación espacial de Tijuana.

Enesesentido, laciudad experimentó una seriede transformaciones asociadas a la modernización neoliberal del país que comenzó a principios de los años ochenta en México. Iniciada luego del agotamiento de la industrialización basada en la sustitución de importaciones y el incremento de su endeudamiento, dicha restructuración económica incluyó la configuración de la región fronteriza norte como una zona de ensamblaje de bajos costos. A través de un régimen fiscal basado en la reducción de las cargas impositivas para los inversionistas extranjeros y el abaratamaniento de la mano de obra, dicha región fue progresivamente industrializada en el marco más amplio del abatimiento del campo mexicano (Katsulis 2008; Rochlin 1997).

Alimentada por la migración permanente de mexicanos expulsados de otras partes del país, principalmente zonas agrícolas, y atraídos por la imagen de una inagotable y permanente prosperidad, entre 1990 y 2005 la población de Tijuana crecio en promedio un $4 \%$ más que el $1.85 \%$ del promedio nacional. $60 \%$ de sus residentes durante este periodo procedieron de otros estados y actualmente el $43.6 \%$ de su población no nació en la ciudad (Instituto Nacional de Estadística y Geografía 2016). En este mismo periodo de tiempo, el territorio de la ciudad se expandió considerablemente. En 1984, por ejemplo, Tijuana cubría 10, 300 hectareas. Para 2005, este número se incrementó hasta 25, 000 hectareas, con una población de 1, 410, 700 habitantes. Con un crecimiento de 2.25 hectareas por día, la ciudad ha más que duplicado su tamaño en 20 años, y para 2005 su zona metropolitana se convirtió en la sexta aglomeración urbana más grande de México.

En el marco de, su ya histórica posición transgresiva y liminar, el flujo constante de individuos en tránsito o buscando asentarse en la ciudad, su expansión territorial sobre todo hacia el este, y el incremento de los índices de criminalidad, han dotado a Tijuana de una ambiguedadeinestabilidadadicionalesaaquellas relacionadas con su origen (Millán 2013). Dicha inestabilidad ha dificultado el establecimiento de límites discernibles que permitan distinguir 
entre dentro y fuera. Es decir, el crecimiento exponencial, vertiginoso y sin freno de la ciudad, sumado a la circulación ilimitada de personas y mercancías, han conferido a su geografía una laxitud y plasticidad, que han puesto a prueba la capacidad de sus habitantes para determinar tanto quien pertence a la ciudad, como el lugar preciso donde ésta termina:

\footnotetext{
J. Mira, está cabrón. El Maclovio Rojas, está bien pinche lejos, es hacia Tecate, pero la verdad no tengo mucha idea de cómo puedes llegar ahí. Lo que necesitas es un carro para llegar ahí, porque en camión si está medio díficil. Además para qué vas para allá, no hay nada que ver ahí y es bien peligroso. Es lo que llaman la "nueva" Tijuana. No estaba ahí hace 15 años y es donde ha llegado toda la gente nueva, del sur sobre todo.

H. ¿Y cuál es la vieja Tijuana, por cierto?

J. Pus esa va del centro de la ciudad donde está la Av. Revolución y la Zona Río, hacia el aeropuerto y de ahí hacia la playa. (Javier, taxista)
}

A la pregunta de cómo llegar al sitio conocido como el ejido Maclovio Rojas, Javier explica lo que aparenta ser el sentido común con respecto al orden urbano en Tijuana, la distribución geográfica de la inseguridad y la expansión del miedo. ${ }^{9}$ Hasta antes de que el crimen desbordara los límites reconocidos por la lógica turística autorizada de la ciudad, el centro de Tijuana, como lo sugieren Arturo y Javier, cumplía la función de fijar y limitar simbólicamente la ambigüedad de la ciudad. Al concentrarse en una franja perpendicular a la frontera, el flujo provocado por la acumulación de actividades transgresoras, pero permitidas en razón de su propia existencia, daba un sentido preciso al orden simbólico y material de la ciudad:

A. El punto es que tu sabías que estabas en Tijuana cuando llegabas a la 'Revu'. De ahí hasta la línea todo era

El Maclovio, como es llamado entre los locales, es el sitio en que poco más de 200 personas fueron disueltas en ácido por quien fue apodado el Pozolero (Vulliamy,2011), permitido. Más allá de ella tampoco es que hubiese mucho que ver. Pero hasta ese punto todos sabíamos que dentro de esos límites todo estaba permitido: beber alcohol, tomar con prostitutas y drogarte si querías. Afuera... pus afuera no había nada que hacer tampoco y además era el tiempo en que si no trabajabas en el aeropuerto o en una maquiladora, no es que tuvieras mucho que hacer en la ciudad. Si había de vez en cuando muertitos, pero todo muy normal. Nada que sorprendiese..$^{10}$

Espacialmente articulado, el orden simbólico de Tijuana aseguraba una cierta consistencia discursiva y práctica. Su crecimiento urbano, demográfico y económico trastocó sin embargo la precaria precisión de esa ordenación. La consecuente expansión de la ciudad, sobre todo hacia el este, así como la densificación del incesante movimiento de personas y mercancías, resultado de la inserción de Tijuana en los circuitos globales de circulación de mercancías e individuos, impuso dificultades prácticas para fijar límites exactos sobre quién pertenece a la ciudad y cualés son las reglas exactas de esa pertenencia:

\begin{abstract}
A. No entiendo qué pasa. Un día te levantas y pum!! Hay una nueva colonia en algún lugar de Tijuana. La verdad yo sé andar muy bien por todas partes, pero sí hay zonas, sobre todo en dirección a Tecate que no conozco. Esa es la gente nueva a qué me refiero y la que está causando todos los pedos. Eso goeyes son de Sinaloa, Michoacán. Del sur pues. No dudo que haya gente trabajadora ahí, pero pus por los menos pagan los más. Y sin manera de saber quién es quién ahí está el problema. ${ }^{11}$
\end{abstract}

La observación de Arturo sobre la expansión de la ciudad hacia el este, en dirección a Tecate, introduce una nota adicional con respecto al

10 El entrevistado se refiere por 'Revu' a la Avenida Revolución que atraviesa el sector turístico y en el que un buen número de bares, clubs nudistas y hoteles se concentran. La línea es propiamente el cruce fronterizo, que se encuentra probablemente a menos de 100 metros de dicha Avenida.

11 Tecate es una ciudad contigua a Tijuana y es parte de su zona metropolitana. Se encuentra situada al este. Pedo es un modo figurado de llamar en este caso a un problema. 
desplazamiento operado en el orden simbólico y material de la ciudad, sobre todo con respecto a la serie de cambios referida arriba.

Como fue dicho previamente, antes de su representacióncomounlugardenarcotraficantes, el orden simbólico de Tijuana descansaba sobre un cierto grado de normalización de la inestabilidad. En el caso estudiado, este orden tiene que ver con la naturalización de la ciudad como un espacio transgresivo e inseguro, pero de límites puntuales dada la distribución territorial de la transgresión, circunscrita sobre todo al centro. La criminalidad en ese sentido, como lo sugieren los relatos de las personas entrevistadas, es siempre experimentada como parte de la naturaleza misma de la ciudad. Lo que cambia al respecto con la caracterízación de Tijuana como una ciudad de narcotráficantes, en el marco de su crecimiento demográfico y territorial, es sin embargo, la ampliación tanto de la identidad, como la localización de los recipientes permanentes de la inestabilidad e inmoralidad que se considera inherente a la ciudad.

En todos los relatos referidos por los habitantes de Tijuana con más de 30 años viviendo en ella, los portadores de la transgresión inherente a la ciudad son las poblaciones de prostitutas y narcos, cuyas identidades son asociadas con actividades ilícitas y que se concentran en el centro de la ciudad. Ello permite fijar espacial y corporalmente, con relativo éxito en lugares y poblaciones específicas, lo que de otra forma representaría un flujo incesante y sin límites, del riesgo y la inseguridad. Sin embargo, la intensificación contemporánea de los homicidios y secuestros, que corre paralela a la militarización de la frontera (Coleman, 2005; Dunn, 1996), la intervención policiaco-militar del estado mexicano en la ciudad como estrategia contra el narcotráfico (Shirk 2014; Vulliamy, 2011), así como a su expansión territorial y crecimiento demográfico, hicieron estallar la comprensión del orden de la inseguridad como un asunto localizable en algunas poblaciones y restringida al centro de Tijuana. Más importante aún, hicieron de todos sus residentes potenciales responsables y víctimas ante la incapacidad de discernir el origen de la inseguridad.

Esta transición fundamental, ha tenido como consecuencia un cambio sustantivo en el modo en que se configura la identidad política de los residentes de la ciudad, así como la manera en que el poder del estado es ejercido en ese espacio urbano. En la siguiente sección serán expuestas algunas consideraciones sobre dicha configuración.

\section{Vulnerabilidad ilimitada: la ciudad de las víctimas}

E. Qué pasó en Tijuana?

Y. Nunca lo entenderías. Nosotros nos hacemos esa pregunta que se agrava todos los días cuando tienes que responder por qué aquellos que tienen que ayudarte no lo hacen. La policía y los malandros son lo mismo. Cuando denuncié el secuestro de mi hijo, en lugar de ayudarme, me pusieron una camioneta todos los días afuera de mi casa, con ellos sentados ahí vigilandome. $Y$ sabes quiénes eran? Los mismos policías que se suponía estaban investigando el caso de mi hijo. Lo que querían era asustarme, hacerme sentir acosada y que pensara que era mejor desistirme de mi acusación.

E. Pero cómo sabe qué eran policías?

Pues porque los había visto en sus oficinas antes (Yolanda, ama de casa).

Hay una serie de apuntes comunes a todos los relatos hasta ahora presentados. El primero de ellos refiere la dificultad para situar el origen de 
la violencia, así como para fijar la identidad de sus ejecutores. La segunda nota en común trata sobre la progresiva ampliación de una sensación de fragilidad y vulnerabilidad, aparejada con el deterioro de las condiciones de seguridad de la ciudad y el anonimato de los perpetradores de la violencia.

Los argumentos oficiales y las versiones mejor establecidas, han asumido que la proliferación de homicidios y secuestros en Tijuana, fue una consecuencia de la fractura interna del cártel del mismo nombre, que como se sugirió arriba, es quien se suponía organizaba el tráfico de drogas en la ciudad, así como controlaba el resto de actividades ilícitas (Alonso 2013; Shirk, 2014; Vulliamy, 2011). La tesis central de esta proposición, basada fundamentalmente en las versiones policiacas oficiales, es que la multiplicación de los homicidios es resultado de la ausencia de un grupo hegemónico que controlase los intercambios entre grupos no estatales armados. La falta de coordinación y la competencia que de ello se desprende, es la condición que incentiva el enfrentamiento armado. En Tijuana como consecuencia, la inseguridad, tal y como asume esta posición, es resultado de una confrontación entre grupos de narcotráficantes opuestos entre sí, en disputa por el territorio de la ciudad como si fuese un campo de batalla.

Esa es la tesis de la guerra por la plaza, matriz central de la posición gubernamental respecto a la comprensión de la guerra contra las drogas de Felipe Calderón, y que obliga naturalmente, siguiendo su proposición central, a intervenir territorialmente para reducir la influencia del narcotráfico (Calderon, 2010; Kenny \& Serrano, 2012). Adicionalmente, al vincular la inseguridad de este espacio urbano, con la amenaza explícita a la seguridad nacional, por la vía de la apropiación del territorio del país con propósitos criminales, Tijuana fue colocada dentro de una política de seguridad más amplia vinculada con la guerra global contra las drogas liderada por Estados Unidos.

En términos locales, esta proposición tiene como sustento principal el incremento de homicidios y la multiplicación de los asesinatos (Molzahn et al. 2012). No obstante, carece de elementos para establecer un contraste empírico que le otorgue certidumbre, al tiempo que simplifica las diferencias regionales $y$ locales a la existencia de una lucha, que en todos los espacios tiene como objeto siempre la misma finalidad: capturar el territorio para hacerlo propicio para la circulación ilimitada de drogas, asi como el control del mercado de consumidores potenciales (Escalante 2012). La tesis de la captura del territorio como objetivo del crimen organizado, obvia la relación compleja ente los objetivos comerciales de grupos criminales, el estado y su respectiva estructura organizacional. No se deduce inmediatamente de la actividad criminal de las organizaciones criminales, que en toda circunstancia aspiren a controlar físicamente territorios particulares. En todo caso la combinación entre objetivos estratégicos, estructuras comerciales y capacidades policiacas estatales, es la que determinan esa posibilidad. No hay manera entonces de determinar con la evidencia disponible (estadísticas e informes oficiales) que en todos los casos sea el control de porciones específicas de territorio el objeto de la disputa, o su razón exclusiva (Escalante 2012; Resa 2014).

Para los residentes de Tijuana dicha claridad es también indemostrable o contradictoria, por lo menos al nivel de la vida diaria. Si los discursos 
sostenidos por las instituciones encargadas de impartir justicia y aplicar la ley en México, insisten sobre la lucha entre narcotráficantes como causa suficiente de la violencia en la ciudad, para los tijuanenses tal distinción ha sido, desde el principio y en términos cotidianos confusa por decir lo menos. Ello ha redundado, como ha sido expuesto arriba en una sensación generalizada de incertidumbre y vulnerabilidad magnificada:

\footnotetext{
P._Antes era diferente. Conocías a tus vecinos y podías confiar en ellos; tanto que las puertas de las casas estaban siempre abiertas, la ciudad prosperaba, e lbamos al 'otro lado' a comprar. Pero hoy se ve cada cosa, mucha gente vagando por la ciudad, sobre todo en el centro, los deportados, los vagabundos, toda la inseguridad y la violencia. No hay forma de distinguir entre dentro y afuera, quién es quién. Todo se mueve. Cuando hicieron el censo en 2010, un montón de gente ya había huído, nadie contestaba a las puertas. Por eso salió mal. Todos estaban escondidos detras de los cientos de rejas que fueron levantadas por todos lados, y detras de las cuales apenas nos sentíamos protegidos. La ciudad se convirtió en una sola reja. (Paty)
}

Alrededor de esta sensación de incertidumbre, reside un miedo generalizado que se desplaza más allá de los límites de la seguridad personal, y se entrelaza con la impresión, igualmente extendida, sobre la incierta y ambivalente relación del estado con la inseguridad en la ciudad. Esta "inseguridad ontológica" (Giddens 1990. Traducción propia), fundada sobre la restructuración de la relación entre el estado como un "guardián oficial" (Traducción propia) y los ciudadanos, describe un estado de ansiedad colectiva y de temor ante lo que se percibe como un movimiento progresivo hacia la desintegración del orden social. Al fondo de este estado, subyace un desplazamiento fundamental que refiere al modo en que los individuos son incluidos dentro del orden político de la ciudad de Tijuana, y cómo son, de acuerdo con ello, tratados y conducidos.
Debajo y alrededor de las experiencias de los tijuanenses con respecto a la guerra contra las drogas, se manifiesta una fuente de incertidumbre y vulnerabilidad, productora de una experiencia distintiva de la ciudad, al tiempo que una forma de identificación emergente de los residentes de la ciudad, consecuente con ella:

\begin{abstract}
Nos volvieron victimas a todos. No porque nos hubiera pasado algo a cada uno, sino porque cada día sentías que la muerte se acercaba a ti. Una o dos casas más allá de la mía se metieron unos encapuchados y sacaron arrastrando al esposo e hijo de una vecina. Dizque eran policías los que lo hicieron... o al menos así se presentaron. Nunca más supimos de ellos, la esposa sigue buscando, pero todo lo que ha encontrado es un muro de negligencias. Ni la policía ni el gobernador le dan respuestas y todos se han hecho pendejos. Es ahí donde dices: esa es la medida de la muerte, dos pinches casas de distancia. Dos casas nada más. Pero igual si salías a la calle te podía tocar una balacera o que te confundieran, o que te secuestraran nomás porque sí. No había razones en realidad. Nunca nadie salió en la tele o en el radio a decir porque estaba pasando esto. Que era el muletas y el cartel de Tijuana contra el de Sinaloa, pero ni así se entiende nada. $Y$ cuando piensas que ni siquiera en tu casa estas seguro entonces te das cuenta del tamaño del desorden. Yo dejé de salir, pero a quien le preguntes te dirá lo mismo. Todo se rompió y preferimos quedarnos adentro aunque tampoco fuera seguro. ${ }^{12}$ (Rebeca)
\end{abstract}

La impresión de Rebeca acerca de la multiplicación de los puntos de contacto entre la vulnerabilidad de los cuerpos de los habitantes de la ciudad, y la creciente inseguridad dentro de la misma, atraviesa todos los relatos disponibles en el contexto previo a la pacificación de la ciudad. Una sensación generalizada de desamparo, angustia y vulnerabilidad absoluta, que tiene como eje articulador la expansión de la inseguridad más allá de los espacios públicos y dentro mismo de los hogares de los tijuanenses.

Pendejo refiere a alguien tonto, en este contexto a quien no ha asumido su responsabilidad. Pinche se utiliza como término derogatorio para designar algo despreciable. 
Este tránsito de la violencia a los espacios íntimos, observa un cambio fundamental en la economía política de la violencia e inseguridad inherentes al origen de la ciudad. La intrusión de la violencia en los hogares de los tijuanenses, demarca un punto que parece sin retorno. Este punto señala la degradación de lo que parecía la última barrera entre el desorden de la ciudad y los cuerpos sin protección de los residentes de Tijuana. Como sostiene Weizman (2007: 210), las paredes del hogar materializan el imperio de la ley y fundan la separación entre lo público y lo privado que hacen posible el orden político, la ley, y la ciudad misma. La depredación de los hogares de los Tijuaneses, cualquiera fuese el origen, observa el deterioro sustantivo de los límites entre lo público y lo privado, que es en realidad, la degradación del order de la ciudad por la acción de una violencia cuyo orígen no es precisable. En ese contexto, las figuras de autoridad pública, como sugiere Rebeca no parecen distinguirse o guardar una distancia, que permita seguir pensando en la oposición entre estado y crimen como fundadora del derecho y de la existencia misma de la ciudad.

En ese escenario lo que acontece es sobre todo, como lo describe Paty, un retraimiento de quienes habitan Tijuana hacia sus hogares, aún si éstos se manifiestan absolutamente precarios en su capacidad de brindar protección y contener la violencia que ocurre en el exterior. Físicamente, la vulneraribilidad de los cuerpos y el espacio doméstico ha intentado ser disminuida, primero, a través de la implementación de barreras físicas para asegurar la separación entre el exterior amenazante y el interior de los hogares (Enriquez 2007). Imponiendo formas de auto-segregación y confinamiento, la expansión del crimen en Tijuana asociada a la guerra contra las drogas modificó de ese modo las interacciones sociales entre sus habitantes, así como el paisaje de la ciudad. En segundo término, a través de la aceptación tácita de la intervención policíacomilitar realizada en nombre de la pacificación de la ciudad.

En tanto el miedo y la desconfianza imperan como motivos fundamentales para la existencia de los tijuanenses, el uso de los espacios públicos así como la participación política se han restringido significativamente a sus minimas expresiones (votar por ejemplo). Ello no ha si no fortalecido las posibilidades de ser vulnerado, al incrementarse la desconfianza entre quienes residen en la ciudad, no existir o crearse mecanismos que compensen la incertidumbre, ni se demanden formas de transparencia que permitan escrutar y limitar la actividad de los agentes estatales encargados de la aplicación de la ley.

En términos del tipo de subjetividad política producida en este contexto, ha emergido una ciudadanía precaria limitada en dos sentidos convergentes. El primero concierne a la restricción voluntaria del movimiento propio y el confinamiento, igualmente voluntario, por temor a ser victimizado. Ello implica directamente una reducción de la vida pública, así como de la apertura que se supone es una de las caraterística centrales de la vida política moderna (Caldeira 2000; Davis 1990). El segundo sentido tiene que ver con la ampliación de una forma de vulnerabilidad ilimitada, por la acción irrestricta de la violencia criminal, y lo que por el momento puede ser caracterizado como una omisión letal por parte de los aparatos de seguridad de estado, incapaces -o complicesde identificar y detener a los perpetradores de dicha violencia (Resa 2016). Sea cual sea el caso, las condiciones bajo las que se vive en Tijuana 
son indicativas de las coordenadas socioespaciales, en que la democracia mexicana es practicada en el contexto de la guerra contra las drogas-

\section{Conclusión}

La proximidad de Tijuana con la frontera con Estados Unidos y su posición dentro de los circuitos globales de producción y circulación de mercancías e individuos, le han conferido una posición predominante en la guerra global contral las drogas. Su caso es ilustrativo de la manera en que esta guerra ha producido condiciones particulares de segregación socioespacial, violencia, inseguridad e incertidumbre. Adicionalmente, el análisis de su funcionamiento como un espacio inseguro, en el contexto de dicha guerra, permite entender la manera en que estas formas específicas de segregación socio-espacial tienen consecuencias políticas fundamentales para la vida democrática en México. De esa manera, bajo las condiciones imperantes de inseguridad y violencia, instigadas por la estrategia antinarcóticos del gobierno mexicano, se han incrementado la incertidumbre e inseguridad, así como la vulnerabilidad de sus ciudadanos.

En este sentido, a través de la exploración de los discursos sobre la identidad de Tijuana como espacio criminogénico, y su ordenamiento como espacio liminar, orientado a la satisfacción de los consumidores estadounidenses, este artículo ha ofrecido evidencia original respecto a la manera en que su representación como un lugar inseguro, se intersecta con el uso que sus ciudadanos hacen de ésta. También por caso, del modo en que dicha representación ha instigado modificaciones espaciales, con un impacto decisivo sobre la manera en que sus residentes circulan e interactúan dentro de Tijuana. En este caso, la guerra contra las drogas, a través de la representación y configuración de Tijuana como un espacio inseguro, ha producido las condiciones necesarias para que sus residentes sean vulnerados por la expansión del miedo e incertidumbre; sumada también a la proliferación de la violencia que ha acompañado el crecimiento demográfico y territorial de la ciudad.

Adicionalmente, el análisis de este caso ha proporcionado evidencia de la manera en la guerra contra las drogas ha producido formas de segregación socio-espacial. Dichas formas, han profundizando sus efectos y generado formas de legibilidad, bajos las que tanto la violencia policiaco-militar es ejercida, como los ciudadanos conducidos. En suma, la exploración de la dimensión socio-espacial de la guerra contra las drogas en México, ha referido la extraordinaria complejidad de su organización y ejercicio, así como las limitaciones inherentes a su práctica.

En el contexto preciso de los análisis sobre la guerra contra los drogas en México y en términos de las investigaciones sobre la geopolítica de lo cotidiano, este artículo contribuye así al desarrollo de un debate sobre los aspectos micro-políticos y espaciales de la prohibición de drogas. En su atención a la percepción del modo en que la violencia es distribuida en la ciudad y los efectos que, sobre los usos del espacio urbano esto tiene, pueden ser sugeridos algunos temas para el desarrollo de una agenda sobre la dimensión socio-espacial y cotidiana de la guerra contra las drogas: i) es necesario avanzar sobre la producción de un entendimiento alternativo con respecto al modo 
en que prácticas de intervención policiacas y comunitarias, producen territorializaciones particulares bajo las que la violencia se moviliza y potencia; ii) mayor énfasis es necesario en el análisis de la organización local de la violencia, el crimen y los mercados ilícitos; iii) de esta manera, el análisis geopolítico de la vida cotidiana en la guerra contra las drogas, tendría que desplazarse principalmente hacia dos aspectos escasamente estudiados. El primero de ellos concierne al análisis del modo en que las prácticas policiacas, son desarrolladas sobre el terreno y su intersección con los imaginarios geopolíticos que subyacen a la guerra contra las drogas. El segundo, refiere al modo en que la guerra contra las drogas ha sido incorporada en la organización del espacio urbano, tanto en lo que hace a la arquitectura de la ciudad, como en la clasificación de sus espacios íntimos. La tarea es urgente, no solo en términos académicos, sino con respecto a las intervenciones gubernamentales que intentan atajar la violencia asociada a la producción y distribución de drogas. Una comprensión cabal de los efectos espaciales que tiene esta guerra, es indispensable para re-orientar el rumbo de una política que se ha mostrado infructuosa, sobre todo en el contexto actual de la reiteración de la militarización de la vida pública en México.

\section{Bibliografía}

Almazán, A. 2013. Chicas Kaláshnikov y Otras Crónicas. México: Océano.

Alonso, G. 2013. "Los años que vivimos horrorizados. Discursos y violencia asociada al narcotráfico en Tijuana 20072010". Fronteras Culturales, alteridad y violencia. Aguilera, M. (Ed.) Tijuana: El Colegio de la Frontera Norte. 115-151.

Astorga, L. 2003. Drogas sin fronteras. México: Grijalbo.

Astorga, L. 2005. El Siglo de las Drogas. México: Plaza y Janes.

Astorga, L. 2015. ¿Qué querían que hiciera? México: Grijalbo. Astorga, L., \& Shirk, D. 2010. "Drug Trafficking Organizations and Counter-Drug Strategies in the U.S.-Mexican Context". Shared Responsibility: U.S.-Mexico Policy Options for Confronting Organized Crime. Olson, E., Shirk, D., \& Selee, A. San Diego, CA: Mexico Institute, Wood- row Wilson International Center for Scholars/Trans-Border Institute.

Berumen, F. 2003. Tijuana la horrible. Entre la historia y el mito. Tijuana, Mexico: El Colegio de la Frontera Norte.

Bezares, H. 2018. Governing the Mexican Drug War: A Political Geography of Public Security and the Organisation of Everyday Violence. PhD diss., Newcastle University, http://theses.ncl.ac.uk/jspui/handle/10443/4164

Boyce, G. A., Banister, J., \& Slack, J. 2015. You and what army? Violence, the state, and Mexico's war on drugs. Territory, Politics, Governance, 3 (4): 446-468.

Boyer, J. 2001. La guerra perdida contra las drogas. México City: Grijalbo.

Bunker, R., \& Sullivan, J. 2010. "Cartel evolution revisited: third phase cartel potentials and alternative futures in Mexico". Small Wars \& Insurgencies: 37-41.

Caldeira, T. 2000. City of Walls: Crime, Segregation, and Citizenship in Sāo Paulo. Berkeley: University of California Press.

Caldeira, T. 1996 "Fortified Enclaves: The New Urban Segregation". Public Culture 8 (2): 303-328.

Calderón, F. 2010. "Sesión de preguntas y respuestas del Presidente Calderón en la comida de clausura del xxiv encuentro nacional de vivienda". Presidencia de la República. 6 de Junio de 2016. Disponible en: http://calderon.presidencia.gob.mx/2010/03/ sesion-de-preguntas-y-respuestas-del-presidente-calderon-enla-comida-de-clausura-del-xxiv-encuentro-nacional-de-vivienda (Consultado en Junio de 2016).

Campbell, H. 2009. Drug War Zone. Austin TX: University of Texas Press.

Campbell, L. 2010. "Los Zetas: operational assessment". Small Wars \& Insurgencies: 55-80.

Chatterjee, I., 2009. Violent morphologies: Landscape, border and scale in Ahmedabad conflict. Geoforum 40 (6):1003-1013

Coleman, M. 2005. "U.S. statecraft and the U.S.-Mexico border as security/economy nexus". Political Geography. 24: 185209.

Davis, M. 1990. City of quartz: Excavating the future in Los Angeles. London: Verso.

Davis, D. 2017. Violence, Fragmented Sovereignty, and Declining State Capacity: Rethinking the Legacies of Developmental Statism in Mexico. En: States in the Developing World. 
Dunn, T. 1996. The Militarization of the U.S.-Mexico Border, 1978-1992. Low-Intensity Conflict Doctrine Comes Home. Austin: University of Texas at Austin.

Enriquez, J. 2007. Entre el miedo y la distinción. La apropiación del espacio público. Los fraccionamientos cerrados en la frontera noroeste de México. Universidad Nacional Autónoma de México, Facultad de Ciencias Políticas y Sociales. México: UNAM.

Escalante, F. 2009. "Territorios Violentos". Nexos. Disponible en: http://www.nexos.com.mx/?p=3951 (Consultado en Abril de 2015).

Escalante, F. 2011. "Homicidios 2008-2009. La muerte tiene permiso". Nexos. Disponible en: http://www.nexos.com.mx/?P=le erarticulo\&Article=1943189 (Consultado en Enero de 2015).

Escalante, F. 2012. El crimen como realidad y representación. Mexico City: El Colegio de Mexico.

Felbab-Brown, V. 2011. "Calderón's Caldron Lessons from Mexico's Battle Against Organized Crime and Drug Trafficking in Tijuana, Ciudad Juárez, and Michoacán". Latin America Institute at Brookings. Latin America Institute at Brookings.

Giddens, A. 1990. The Consequences of Modernity. Stanford: Stanford University Press.

Glück, Z., \& Low, S. 2017. "A sociospatial framework for the anthropology of security". Anthropological Theory. 17(3): 281-296.

Glebbeek, M. L. \& Koonings, K. 2015. Between Morro and Asfalto. Violence, insecurity and socio-spatial segregation in Latin American cities. Habitat International, Volume 54, pp. 3-9

González, F. 2009. "Mexico's Drug Wars Get Brutal”. Current History. (108): 72-76.

Gregory, D. 1994. Geographical Imaginations. Oxford: Blackwell.

Gregory, D. 1995. "Imaginative Geographies". Prog Hum Geogr. 19: 448-485.

Gregory, D. 2011. "The everywhere war". The Geographical Journal. 238-250.

Instituto Nacional de Estadística y Geografía. 2016. Encuesta Nacional de Seguridad Pública Urbana (ENSU). Disponible en: http://www.beta.inegi.org.mx/proyectos/enchogares/regulares/ ensu/

Katsulis, Y. 2008. Sex, Work and the City: The Social Geography of Health and Safety in Tijuana, Mexico. Austin, TX: University of Texas Press.

Kenny, P., \& Serrano, M. 2012. México's Security Failure: Collapse into Criminal Violence. New York: Routledge.

Knowles, C. 2000. Bedlam on the Streets. London/New York: Routledge.

Koonings, K. \& Kruijt, D. 2007. Fractured cities. Social exclusion, urban violence and contested spaces in Latin America. London/New York: Zed Books.

Koonings, K. \& Kruijt, D. 2009. Mega-Cities: The Politics of Urban Exclusion and Violence in the Global South, London: Zed Books

Legg, S. 2005. "Foucault's Population Geographies: Classifications, Biopolitics and Governmental Spaces".
Population, Space and Place. (11): 137-156.

Legg, S. 2007. Spaces of Colonialism: Delhi's Urban Governmentalities. Malden, USA: Blackwell Publishing.

López, J. 2014. "Tijuana Merece ser Curada. Intervenciones Socio-culturales del gobierno y la sociedad frente a la crisis de inseguridad 2006-2012". COLEF3, Estudios Regionales. Tijuana: COLEF.

Mendoza, N. 2008. Conversaciones del desierto: cultura, moral y tráfico de drogas. México City: CIDE.

Millán, O. 2013. Viajes al Este de la Ciudad. Una Crónica de la Guerra contra el Narco en Tijuana. México: TRILCE/CONACULTA.

Molzahn, C., Ríos, V., \& Shirk, D. 2012. Drug Violence in Mexico. Data Analysis Through 2011. San Diego CA.: TransBorder Institute/University of San Diego.

Morales, M. 2012. "La Lucha por la Seguridad en México": Operativos, Homicidios y Crimen Organizado entre 2007 y 2010. Colegio de México, Centro de Estudios Demográficos Urbanos y Ambientales. México: COLMEX.

Morales, M. 2013. "Factores que explicaron los niveles de homicidios y fallecimientos por rivalidad delincuencial entre 2007 y 2010 en México". Coyuntura Demográfica. (3): 17-22.

Paley, D. 2014. Drug War Capitalism. Oakland: AK Press.

Resa, C. 2014. "Mercados Locales de Violencia en México". Recuperado de Carlos Resa Nestares: http://www.uam.es/ personal_pdi/economicas/cresa/LocalViolence0613.pdf

Resa, C. 2016. "No, la culpa de la violencia en México no fue del gobierno de Calderón". Recuperado de Carlos Resa Nestares: http://www.uam.es/personal_pdi/economicas/cresa/nota0816.pdf

Rochlin, J. 1997. Redefining Mexican "Security". Society, State, and Region under NAFTA. Colorado: Lynne Rienner Publishers.

Rodgers, D. 2006. "The State as a Gang. Conceptualizing the Governmentality of Violence in Contemporary Nicaragua". Critique of Anthropology. 26 (3): 315-330.

Romero, A. 2012. Análisis de la Economía del Crimen en el Espacio Intraurbano de Tijuana, Baja California. Colegio de la Frontera Norte. Tijuana: Colegio de la Frontera Norte.

Rose, N. 2004. Powers of Freedom. Reframing political thought. Cambridge: Cambridge University Press.

Ruiz, R. 1998. On the Rim of Mexico. Encounters of the Rich and Poor. Boulder, Colorado: Westwiew.

Serrano, M. 2012. "State-crime relations in México". Violence, Coercion, and State-Making in Twentieth-Century Mexico the Other Half of the Centaur. Pansters, W. Standford, CA: Standford University Press. 263-307.

Shirk, D. 2014. "A Tale of Two Mexican Border Cities: The Rise and Decline of Drug Violence in Juárez and Tijuana". Journal of Borderlands Studies. 29 (4): 481-502.

Shirk, D., Wood, D., \& Olson, E. 2014. Building Resilient Communities in Mexico: Civic Responses to Crime and Violence. Woodrow Wilson International Center for Scholars, Mexico Institute. Washington DC: Woodrow Wilson International Center for Scholars. 
Shirk, D., \& Wallman, J. 2015. “Understanding Mexico's Drug Violence". Journal of Conflict Resolution. 59 (8): 1348-1376.

Vulliamy, E. 2011. Amexica. War along the Borderline. London: Vintage.

Watt, P., \& Zepeda, R. 2012. Drug War Mexico: Politics, Neoliberalism and Violence in the New Narcoeconomy. New York: Zed Books.

Womer, S., \& Bunker, R. 2010. "Sureños gangs and Mexican cartel use of social networkings". Small Wars \& Insurgencies. 81-94.
Wright, M. 2011. Necropolitics, Narcopolitics, and Femicide: Gendered Violence on the Mexico-U.S. Border. Signs, 36 (3): $707-$ 731.

Wright, M. 2013. Feminicidio, narcoviolence, and gentrification in Ciudad Juárez: the feminist fight. Environment and Planning D: Society and Space, Volume 31: 830-845

Yeh, R. 2017. "Visas, Jokes, and Contraband: Citizenship and Sovereignty at the Mexico-U.S. Border". Comparative Studies in Society and History. 59 (1): 154-182. 